\title{
A sensitivity based methodology for optimal placement of distributed generation in meshed power systems
}

\begin{abstract}
Distributed generation (DG) plays a considerable role in minimizing power losses in realworld electric power systems. This benefit can be optimally achieved by determining the optimum placement of DG units. The erroneous placement of DG units in electric power networks can lead to high power loss increment and results in lower limit violation of load buses voltages. Hence, this paper suggests a sensitivity-based procedure for the optimal placement of DG in meshed power systems using suitable technologies. The efficacy of minimizing real power loss is considered as a key criterion. The ranking of candidate locations is determined based on two types of active power loss sensitivities along with loss reduction rates that are obtained by different types and capacities of DGs. A correlation parameter is proposed and suitable weighting factors are defined for each sensitivity type, DG type, and DG size. A priority list that includes both the sensitivity indexes of real power loss and used pragmatic indicators is estimated to rank the optimal locations for DG units placement. Moreover, a sorting index is deduced to identify the appropriate DG type(s) for each candidate site. Three rife DG types are used in this paper. The IEEE 30-bus test system is selected to carry out the proposed methodology. Results show that the estimated priority index can attain the best active loss reduction rates. Several buses can be safely precluded as candidate sites using the proposed methodology. The approach can reduce the computational process of identifying the optimal locations for DG placement. Moreover, results point out that certain locations can be selected to set more than one DG type and considerably minimize active power losses instead of accommodating several DG units at differentsites with one DG type.
\end{abstract}

Keyword: Distributed generation; Sensitivity analysis; Power losses reduction; Meshed power systems 